\title{
Glaucoma in High Myopia
}

\author{
Jost B. Jonas, Songhomitra Panda-Jonas, \\ and Kyoko Ohno-Matsui
}

\section{Key Points}

- In all highly myopic eyes, glaucomatous optic neuropathy may specifically be ruled out, in attempt not to miss the diagnosis

- High axial myopia is a major risk factor for glaucomatous or glaucomalike optic neuropathy, even at a normal intraocular pressure.

- Morphological risk factors for glaucoma in high myopia may be an enlarged optic disc and enlarged parapapillary delta zone.

- Histological hallmarks of the highly myopic optic nerve head are an elongated and thinned lamina cribrosa, steepened trans-lamina cribrosa pressure gradient, enlarged optic disc, elongated peripapillary scleral flange (as equivalent of parapapillary delta zone), and a shallowing of the physiological optic cup.

\subsection{Introduction}

Axial myopization leads to marked changes in the morphology of the posterior ocular segment including the anatomy of the optic nerve head. The changes of the optic nerve head include an enlargement of all three layers of the optic disc (i.e., optic disc Bruch's membrane opening, optic disc choroidal opening, and optic disc scleral

\footnotetext{
J. B. Jonas $(\bowtie) \cdot$ S. Panda-Jonas

Department of Ophthalmology, Medical Faculty Mannheim, Ruprecht-Karls-University Heidelberg, Mannheim, Germany e-mail: jost.jonas@medma.uni-heidelberg.de

K. Ohno-Matsui Department of Ophthalmology and Visual Science, Tokyo Medical and Dental University, Tokyo, Japan
} 
opening) with the development of a secondary macrodisc, and an enlargement and shallowing of the cup. Further morphological changes are an elongation and thinning of the lamina cribrosa with a secondary reduction in the distance between the intraocular space with the intraocular pressure (IOP) and the retro-lamina compartment with the orbital cerebrospinal fluid pressure, and a direct exposure of the peripheral posterior lamina cribrosa surface to the orbital cerebrospinal fluid space. In the parapapillary region, an elongation and thinning of the peripapillary scleral flange with development and enlargement of the parapapillary gamma zone (defined as the parapapillary Bruch's membrane free region) and delta zone (defined as the region between the optic disc border and the merging line of the optic nerve dura mater with the posterior sclera) occurs, in addition to an elongation and thinning of the peripapillary border tissue of the choroid (Jacoby). The optic rotates around the vertical axis, and less often and to a minor degree around the horizontal axis und the sagittal axis. These changes make it more difficult to differentiate between myopic changes and (additional) glaucoma-associated changes such as a loss of neuroretinal rim and thinning of the retinal nerve fiber layer, and these changes may make the optic nerve head more vulnerable potentially explaining the increased prevalence of glaucomatous optic neuropathy in highly myopic eyes.

\subsection{Anatomy of the Optic Nerve Head in High Myopia}

The anatomy of the optic nerve head in primary high myopia is markedly influenced by the axial elongation. This process of sagittal enlargement of the myopic globe takes place predominantly in the posterior hemisphere of the eye, and the changes are more pronounced the closer to the posterior pole [1-5]. Histological studies have shown that with longer axial length, the axial elongation-associated thinning of the sclera was most pronounced close to the posterior pole, while the scleral thickness in the pars plana region did not vary significantly between eyes of various axial length. In a similar manner, the thinning of the choroid was more marked the closer to the macula [6].

It has been postulated that the main driver for the scleral and choroidal thinning in the process of axial elongation may be a new production and elongation of Bruch's membrane in the equatorial and retroequatorial region of the eye [7]. Findings leading to this hypothesis were the following:

- the volume of the scleral and choroid did not markedly increase after the end of the second year of life and that both parameters were independent of axial length [3, 5];

- the thickness of Bruch's membrane was not associated with axial length so that its volume increased in the process of axial myopization [8];

- the main determinant of the process of emmetropization (and myopization) is the length of the optical axis which ends at the photoreceptor outer segments and not at the scleral posterior surface;

- the structure with a minimum of biomechanical strength and being located closest to the photoreceptor outer segments is Bruch's membrane;

- a biomechanical study revealed that the stiffness of Bruch's membrane was comparable or higher than those of other ocular tissues and that it could sustain a relatively high pressure of $80 \mathrm{mmHg}$ or higher before rupture [9]; 
- the density of the retinal pigment epithelium cells and the retinal thickness decreased in the midperiphery with increasing axial length, while both parameters, measured in the macular region, were independent of axial length $[10,11]$; and

- the optic disc-fovea distance increased with longer axial length by the development and enlargement of parapapillary gamma zone while the length of Bruch's membrane in the macular region was independent of axial length [12-14].

These anatomical findings and the postulated mechanism of Bruch's membrane enlargement in the midperiphery as the driver for the cylindrical-like sagittal enlargement of the globe are associated with typical myopic changes in the anatomy of the optic nerve head. These include the following: an enlargement of the optic nerve head with the development of a secondary or acquired macrodisc $[15,16]$; enlargement and shallowing of the optic cup, so that the spatial contrast between the height of the neuroretinal rim and the depth of the optic cup is decreased; elongation and thinning of the lamina cribrosa [17, 18]; development and enlargement of parapapillary gamma zone and delta zone $[19,20]$; and rotation of the optic disc mostly around the vertical axis [21,22].

The enlargement of the optic disc (as defined by the peripapillary ring) affects all three optic disc layers: Bruch's membrane opening; the choroidal opening, in which the choroid is separated from the intrapapillary compartment by the peripapillary border tissue of the choroid (Jacoby); and the scleral opening which is spanned by the lamina cribrosa. The (passive) enlargement of all three optic disc layers may be due to the finding that the myopic axial elongation additionally includes a widening of the eye in the horizontal and vertical directions [23]. For each millimeter increase in axial length, both the horizontal globe diameter and the vertical globe diameter increase by approximately $0.2 \mathrm{~mm}$ [23]. Since the primary and active enlargement of Bruch's membrane may occur in the equatorial region, an increased tension within Bruch's membrane and within the choroid and the sclera may develop at the posterior pole. It would lead to a stretching of the openings of all three optic disc layers. It has been discussed that if the increased tension within Bruch's membrane is not sufficiently released by the enlargement of the physiological Bruch's membrane opening in the optic disc, secondary defects in Bruch's membrane may develop starting with the formation of lacquer cracks and ending the formation of macular Bruch's membrane defects [24-27]. If the enlargement of the optic disc Bruch's membrane opening is more marked than the enlargement of the optic disc choroidal opening, a part of the parapapillary region will no longer be covered by Bruch's membrane, so that parapapillary gamma zone as Bruch's membrane-free region develops [19, 20]. The uneven enlargement of the opening of the various layers of the optic disc may thus be one of the reasons for the development of parapapillary gamma zone.

The enlargement of the optic disc in axially elongating eyes leads secondarily to an enlargement of the optic cup, while the shape of the neuroretinal rim may still follow the so-called ISNT (inferior-superior-nasal-temporal) rule, with the small rim part being located usually in the temporal horizontal disc region [28]. Due to the stretching and enlargement of the optic disc and cup, the spatial contrast between the height of the neuroretinal rim and the depth of the optic cup appears to diminish, so that it becomes clinically more difficult to delineate the optic cup form the neuroretinal rim. 
The enlargement of the opening in the scleral layer of the optic disc is associated with a stretching and thinning of the lamina cribrosa $[17,18]$. Since the diameter of the retrobulbar optic nerve is not directly affected by the process of axial elongation, the myopic enlargement of the posterior surface of the lamina cribrosa has as consequence that a peripheral annular-like region of the lamina is no longer covered or buffered by the solid optic nerve but is directly facing the orbital cerebrospinal fluid space.

Outside of the optic disc border, parapapillary gamma zone and delta zone develop during axial elongation (Fig. 11.1) [19-21, 24, 29-35]. Gamma zone has been defined as the Bruch's membrane-free zone at the optic disc border. It is usually larger on the temporal side than on the nasal side. Prevalence and size of gamma zone is strongly associated with myopic axial elongation and the amount of the optic disc rotation around the vertical axis [21]. Two mechanisms may lead to the development of gamma zone. As pointed out above, gamma zone may develop if the enlargement of the optic disc Burch's membrane opening is more marked than the enlargement of the optic disc choroidal opening. A second mechanism may be connected with a shift of the three optic disc layers during axial elongation. One may assume that at birth all three optic disc layers are aligned to each other. If in the process of axial elongation Bruch's membrane is newly formed and enlarged in the equatorial region, the optic disc Bruch's membrane opening may shift in direction to the macula. If this movement is less marked for the optic disc choroidal layer and scleral layer than it is for the Bruch's membrane opening, a misalignment of the layers will occur, with the Bruch's membrane opening being located most to the macular side and the scleral opening being located most to the nasal side. This

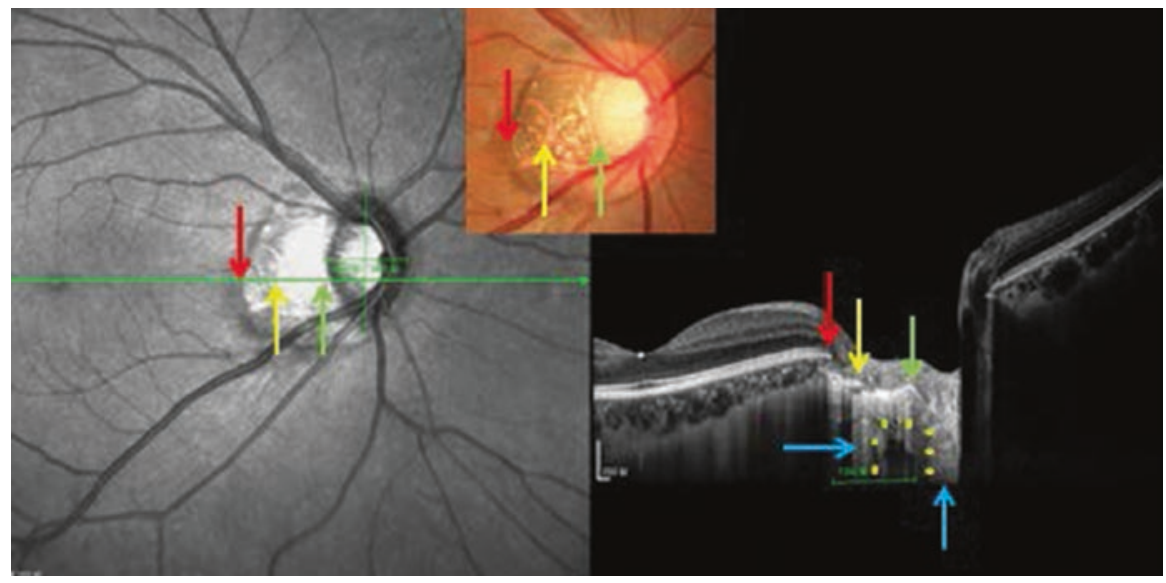

Fig. 11.1 Optical coherence tomographic image of the optic nerve head, showing parapapillary gamma zone (between red arrow and yellow arrow) with Bruch's membrane being absent, and parapapillary delta zone (between yellow arrow and green arrow (optic disc border)) showing an elongated peripapillary scleral flange as anterior border of the orbital cerebrospinal fluid space (yellow stars) between the optic nerve dura mater (horizontal blue arrow) and the optic nerve with its surrounding pia mater (vertical blue arrow) 
phenomenon would explain the overhanging of Bruch's membrane on the nasal side of the optic disc in moderately myopic eyes, and it could be a second mechanism for the development of parapapillary gamma zone [36]. It would also explain the paradoxical course of the optic nerve through the optic nerve head channel with the optic nerve entering the canal from nasal anteriorly and reaching the vitreous compartment temporal posteriorly, although the optic nerve arrives at the eye globe coming from the posterior part of the orbit.

Parapapillary delta zone is located at the optic disc border within gamma zone and has been defined as the region of the elongated and thinned peripapillary scleral flange $[19,20]$. The peripapillary scleral flange is the continuation of the inner half of the posterior sclera and continues into the lamina cribrosa, separated from the latter by the peripapillary scleral border tissue of Elschnig [37, 38]. It forms the anterior end of the orbital cerebrospinal fluid space. In axially elongated eyes, in particular beyond an axial length of $26.5 \mathrm{~mm}$, the peripapillary scleral flange gets markedly elongated and thinned [37, 39]. It may change its dimensions from a normal thickness and length of about $0.5 \mathrm{~mm}$ to a length of $5 \mathrm{~mm}$ and a thickness of less than $100 \mu \mathrm{m}$. The ophthalmoscopical delineation between gamma zone and delta zone can be facilitated by searching for the peripapillary arterial circle of Zinn-Haller which is usually located at the merging line of the optic nerve dura mater with the sclera, i.e., at the peripheral end of delta zone [40, 41].

The optic disc shape as perceived upon ophthalmoscopy is normally slightly oval $[42,43]$. It has to be taken into account that the ophthalmoscopically determined optic disc shape is different from the true optic disc shape, since the location of the optic disc close to the nasal side leads to perspective distortion, usually resulting in a perspective shortening of the horizontal disc diameter [44]. In addition, the optic disc undergoes rotational changes in the process of myopic axial elongation [22]. These are mainly an optic disc rotation around the vertical axis, and to a minor degree a rotation around the horizontal axis and the sagittal axis. A rotation round the vertical axis leads to a perspective shortening of the horizontal disc diameter, a rotation round the horizontal axis leads to a perspective shortening of the vertical disc diameter, a rotation round the sagittal axis does not change the perspective appearance of the disc diameters [44]. The rotation around the vertical axis is associated with the development and enlargement of gamma zone at the temporal side, and a rotation around the horizontal axis is associated with the development and enlargement of gamma zone at the inferior border of the optic disc. These optic disc rotations explain the ophthalmoscopical appearance of the markedly vertically oval shape of optic discs in highly myopic eyes $[15,16]$. The difference between the twodimensional ophthalmoscopical optic disc shape and the optic disc shape as determined three-dimensionally by optical coherence tomography increases with longer axial length, due to the progressing disc rotation around the vertical axis [44]. It has remained elusive which forces lead to the disc rotation around the vertical axis in progressive axial myopia. One reason could be, as outlined above, the increasing misalignment of the three optic disc openings, with the Bruch's membrane opening moving in temporally and the scleral opening staying partially behind. The resulting overhanging of Bruch's membrane into the nasal part of the optic disc channel leads 
to a shortening of the effective horizontal disc diameter. A second mechanism could be a backward strain or pull of the optic nerve dura mater on the peripapillary region $[45,46]$. In a recent study, Demer and colleagues proposed that the backward pull of the optic nerve on the optic nerve head in markedly axially elongated eyes could lead to a novel mechanical load on the globe at the optic nerve head in ocular adduction [ 45]. Since the optic nerve originates in the nasal superior region of the orbit and inserts in the posterior nasal region of the eye, adduction of the globe would lead in axially elongated eyes to a backward pull of the optic nerve on the temporal and temporal inferior side of the optic nerve head. Such a mechanism could explain the marked rotation of the optic nerve head mainly around its vertical axis (and a slight rotation around its horizontal axis with a backward movement of the inferior optic disc pole) in highly elongated eyes. Since the optic nerve dura mater as compared to the pia mater or the neural tissue of the optic nerve may be biomechanically the strongest element, one may assume that the traction is forwarded by the optic nerve dura mater to its insertion on the globe, i.e., the merging line of the dura mater with the posterior sclera or the peripheral end of the peripapillary scleral flange. Traction on the merging line could also lead to an elongation of the peripapillary scleral flange and indirectly to the development and enlargement of parapapillary gamma zone. The study by Demer has been confirmed and extended by investigations conducted by Wang and colleagues who found that that the optic nerve head strains due to the pull by the optic nerve dura mater on the peripapillary sclera during a lateral eye movement of $13^{\circ}$ were as high as, or higher than, those resulting from an IOP of $50 \mathrm{mmHg}$ [46]. Fitting with the notion of a biomechanical role the optic nerve may play for the anatomy and physiology of the optic nerve head is the finding of peripapillary suprachoroidal cavitations which are located mostly in the inferior peripapillary region in about $17 \%$ of highly myopic eyes [47-50]. Their development may also be explained by a backward pull of the optic nerve dura mater on the temporal and inferior peripapillary sclera. Correspondingly, peripapillary suprachoroidal cavitations are associated with an optic disc rotation around the vertical disc axis and high axial myopia [50].

The cutoff for the differentiation between moderate myopia and high myopia at which the axial elongation associated enlargement of the optic nerve head starts has not concisely been assessed so far. According to hospital-based investigations and population-based studies, the optic disc and parapapillary gamma zone start to increase at about a value of approximately -8.00 diopters or an axial length of about $26.5 \mathrm{~mm}[51,52]$. At the same cutoff values, the prevalence of myopic retinopathy increases [53-55].

\subsection{Increased Prevalence of Glaucoma in High Myopia and Associated Factors}

Population-based investigations and hospital-based studies have shown that the prevalence of glaucomatous optic neuropathy (GON) was higher in highly myopic eyes than in emmetropic eyes [53, 56-69]. To cite an example, in the Beijing 
Eye Study the prevalence of GON was significantly higher in eyes with a myopic refractive error exceeding -6 diopters than in the remaining eyes, while between the hyperopic eyes, the emmetropic eyes and the eyes with low to moderate myopia (myopic refractive up to -6 diopters or less), the frequency of glaucoma did not vary significantly [53]. Since the IOP pressure did not differ significantly between the highly myopic eyes and the remaining eyes, it suggested a higher glaucoma susceptibility in the highly myopic eyes. In the Blue Mountains Eye Study, the glaucoma prevalence was higher in moderate to high myopes $(4.4 \%)$ than in emmetropes (1.5\%) [63]. In the Barbados Eye Study, myopia increased the odds of having glaucoma while hyperopia reduced it [61]. In the Malmö eye survey on more than 30,000 individuals, the glaucoma prevalence increased with increasing myopia, and the association between myopia and glaucoma was strong at lower IOP levels, and weakened gradually with increasing IOP [64, 67]. The reason for a discrepancy between studies on an association between myopia and glaucoma may be that not all studies differentiated between moderate myopia, which may not be associated with an increased glaucoma prevalence, and high myopia with an increased frequency of GON [65]. Accordingly, in the Beijing Eye Study the low to moderate myopic group, the emmetropic group and the hyperopic group did not vary significantly in the prevalence of glaucoma in the present study [53]. By the same token, in non-highly myopic individuals, inter-eye differences in refractive error were not significantly correlated with inter-eye differences in neuroretinal rim area and mean visual field defect nor were the neuroretinal rim area, the horizontal and vertical cup/disk diameter ratios and the mean visual field loss correlated with refractive error in an interindividual statistical analysis [65].

A previous study revealed that at a given IOP in patients with chronic openangle glaucoma, the amount of optic nerve damage was more marked in highly myopic eyes with large optic discs than in non-highly myopic eyes [68]. This observation was supported by a hospital-based study in which Nagaoka and colleagues examined 172 patients with a mean axial length of $30.1 \pm 2.3 \mathrm{~mm}$ (range: 24.7-39.1 mm) and in which the prevalence of GON (overall: 28\%) was 3.2 times higher $(P<0.001)$ in large optic discs $\left(>3.79 \mathrm{~mm}^{2}\right)$ than in normal-sized discs or small discs $\left(<1.51 \mathrm{~mm}^{2}\right)$ after adjusting for older age. Interestingly, axial length was not significantly $(P=0.38)$ associated with glaucoma prevalence in that model [70]. It suggested that the axial elongation-associated enlargement of the optic disc as compared to the axial elongation itself was one of the main factors for the increased glaucoma susceptibility in the highly myopic eyes. In a following study, it was additionally detected that an enlarged parapapillary delta zone, together with an enlarged optic disc, was associated with the increased glaucoma prevalence in highly myopic eyes, while in the multivariate analysis, parapapillary gamma zone was not related with the glaucoma prevalence [71]. In that study on 519 eyes with a mean axial length of $29.5 \pm 2.2 \mathrm{~mm}$, prevalence of GON increased from $12.2 \%$ in the group with an axial length of $<26.5 \mathrm{~mm}$ to $28.5 \%(24.4,32.5)$ in the group with an axial length of $\geq 26.5 \mathrm{~mm}$, to $32.6 \%$ in the group with an axial length of $\geq 28 \mathrm{~mm}$, to $36.0 \%$ in the group with an axial length of $\geq 29 \mathrm{~mm}$, and to $42.1 \%$ in eyes with an axial length of $\geq 30 \mathrm{~mm}$. 


\subsection{Potential Reasons for the Association Between Glaucoma and High Myopia}

The main factors associated with the increased prevalence of GON in the highly myopic eyes were the size of the optic disc and the prevalence and size of parapapillary delta zone. Histomorphometric studies have revealed that the axial elongation-associated enlargement of the optic disc is associated with an elongation and thinning of the lamina cribrosa. Since the lamina cribrosa is the functional border tissue between the intraocular compartment with the IOP and the retrobulbar compartment with the orbital cerebrospinal fluid pressure, a thinning of the lamina cribrosa leads to a decreased distance between both compartments and to a steepening of the trans-lamina cribrosa pressure gradient between both compartments [17, 18]. An elevated the trans-lamina cribrosa pressure difference or a steepening of the trans-lamina cribrosa pressure gradient have been discussed to be associated with the glaucomatous damage to the retinal ganglion cell axons when passing through the lamina cribrosa [72-76]. The thinning of the lamina cribrosa may thus be one of the reasons for the association between an enlargement of the optic disc size and a higher prevalence of GON. Additional potential reasons for the increased glaucoma susceptibility in highly myopic optic disc may be a rearrangement of the lamina cribrosa pores and the whole lamina cribrosa architecture by the axial elongationassociated stretching of the lamina cribrosa. Theoretically, it may have a similar effect as the scarring of the lamina cribrosa due to the glaucomatous process itself, what may also lead to increased glaucoma susceptibility at a given IOP [77].

Histological studies have additionally shown that the myopic enlargement of the posterior surface of the lamina cribrosa leads to an exposure of a peripheral annular-like region of the lamina directly to the orbital cerebrospinal fluid space [18]. Since the cerebrospinal fluid in contrast to the solid optic nerve tissue cannot resist a local backward bowing of the lamina cribrosa, the exposure of the posterior lamina cribrosa surface to the cerebrospinal fluid space may allow the development of acquired pits of the optic nerve head which are typically located at the optic disc border.

The peripapillary scleral flange is the biomechanical anchor of the lamina cribrosa to the posterior sclera, with both tissues being partially separated from each other by the peripapillary border tissue of Elschnig the scleral flange [38]. In the process of axial elongation, the length of the peripapillary scleral flange can increase by a factor of $10 \times$, and its thickness can get reduced to $10 \%$ of its original value [19, 78]. These marked anatomical changes may have an effect on the biomechanical properties of the flange as the anchor of the lamina cribrosa and may be one of the reasons for the increased glaucoma susceptibility in high myopia. This notion fits with the observation that the size of parapapillary gamma zone, after adjusting for axial length was not significantly associated with the glaucoma prevalence. Gamma is the zone free of Bruch's membrane, and Bruch's membrane, connected with the lamina cribrosa by the thin peripapillary choroidal border tissue of Jacoby may not directly be involved in the biomechanics of the lamina cribrosa in glaucoma.

The lamina cribrosa is nourished by vessels originating from the peripapillary arterial circle of Zinn-Haller, which is located approximately at the merging point 
of the posterior sclera with the dura mater of the optic nerve $[40,41,79]$. The longer the peripapillary scleral flange, the larger is the distance between the arterial circle and the optic nerve head. The axial elongation-associated elongation of the peripapillary scleral flange may thus potentially have importance for the blood supply of the lamina cribrosa and be another factor for an increased susceptibility of a highly myopic disc for optic nerve damage.

It has remained unclear whether the axial elongation-associated enlargement of the optic disc is an independent risk factor for an increased GON susceptibility in high myopia. In non-highly myopic eyes, the optic disc size was not associated with the prevalence and amount of GON [77, 80, 81]. Since the elongation and thinning of the lamina cribrosa and of the peripapillary scleral flange are strongly associated with the optic disc enlargement, one may assume that the disc enlargement is secondarily, and not causally, associated with the increased prevalence of GON in high myopia. It has also remained elusive whether the axial elongationassociated thinning of the peripapillary choroid has an influence on the GON susceptibility [82].

\subsection{Intraocular Pressure and Glaucoma in High Myopia}

In a recent pilot study on 517 eyes with a mean axial length of $29.5 \pm 2.2 \mathrm{~mm}$, the IOP did not differ significantly $(P=0.53)$ between the glaucoma group $(n=141(27.3 \%)$ eyes) and the non-glaucomatous group $(n=376(72.7 \%))$ $(14.5 \pm 3.3 \mathrm{mmHg}$ versus $14.7 \pm 2.5 \mathrm{mmHg})$ [83]. Only in the eyes with an axial length of equal to or less than $27.4 \mathrm{~mm}$, the prevalence of GON was correlated with higher IOP $(P=0.037$; odds ratio $(\mathrm{OR}): 1.35 ; 95 \%$ confidence interval $(\mathrm{CI}): 1.02,1.80)$, while in the eyes with a longer axial length, the prevalence of GON was not significantly $(P=0.97)$ associated with IOP in a multivariate analysis adjusting for age, axial length, shorter vertical diameter of the temporal arterial arcade and longer minimal optic disc diameter. There were major limitations of the study as follows: the diagnosis of GON was based on the ophthalmoscopic appearance of the optic nerve head, without taking into account results of visual field examinations or findings obtained by optical coherence tomography; and the glaucomatous group as compared to the non-glaucomatous group had a significantly higher prevalence of anti-glaucomatous therapy. It could suggest that the lack of a difference in IOP between both groups was due to the higher prevalence of IOP-lowering therapy in the glaucomatous group. In the multivariate analysis with adjustment for the prevalence of anti-glaucomatous treatment however, the IOP was not significantly associated with the glaucomatous versus non-glaucoma group.

If the results of the study are valid, it may not indicate that there is no association between IOP and glaucoma in high myopia, but that highly myopic glaucomatous eyes as compared with non-highly myopic glaucomatous eyes may have a markedly lower IOP threshold to develop optic nerve damage. It could indicate that an IOP of perhaps lower than $10 \mathrm{mmHg}$ might be necessary to prevent the development of GON in these highly myopic eyes, and that in highly myopic eyes with axial 
elongation associated enlargement and stretching of the optic disc and parapapillary region as the main risk factors for GON in high myopia a normal IOP may be sufficient to lead to GON.

\subsection{Therapy of Glaucoma in High Myopia}

Although it has not yet been firmly proven that GON in high myopia is dependent on IOP, and although a randomized trial on the effect of lowering of IOP as therapy of glaucoma in highly myopic eyes has not been performed yet, most researchers recommend lowering IOP in highly myopic patients with glaucoma. Based on the morphological findings described above, the target pressure in highly myopic glaucoma may be lower than in non-highly myopic glaucoma. A factor markedly complicating the clinical situation is the difficulty in detecting the presence of GON and, in particular, in detecting the progression of optic nerve damage in highly myopic glaucoma. Due to the peculiar anatomy of the optic nerve head in highly myopic eyes, most diagnostic procedures fail in precisely assessing the status of the optic nerve in highly myopic eyes with glaucoma. It includes factors such as:

- a decreased spatial and color contrast between the neuroretinal rim and the optic cup making a delineation of both structures more difficult;

- a peripapillary retinoschisis leading to an incorrect segmentation of the retinal nerve fiber layer upon optical coherence tomography;

- a large gamma zone (and delta zone) which makes using the end of Bruch's membrane as reference point for the measurement of the neuroretinal rim useless;

- a large gamma zone also markedly further reduces the anyway relatively low value of parapapillary beta zone as indicator for GON; and

- macular Bruch's membrane defects and other reasons for non-glaucomatous visual field defects which reduces the diagnostic precision of perimetry for the detection of presence and progression of GON.

The mode of therapy of lowering IOP in highly myopic glaucoma is similar to the therapy of open-angle glaucoma in non-highly myopic eyes and includes topically applied drugs reducing the production of aqueous humor and/or increasing the outflow of aqueous humor, laser procedures aimed at the trabecular meshwork to increase aqueous humor outflow, and surgical procedures for increase of aqueous humor outflow or reduction of aqueous humor production. If fistulating procedures, such as trabeculectomy, are performed, one may have to take into account a potentially increased risk of postoperative choroidal swelling and detachment and even expulsive hemorrhage. Potential reasons might be the marked thinning (compression) of the choroid in highly myopic eyes and an oblique course of the short posterior ciliary arteries (and potentially vortex veins) through the sclera in highly myopic eyes, while in emmetropic eyes the short posterior ciliary arteries run almost perpendicularly through the sclera (Fig. 11.2). 


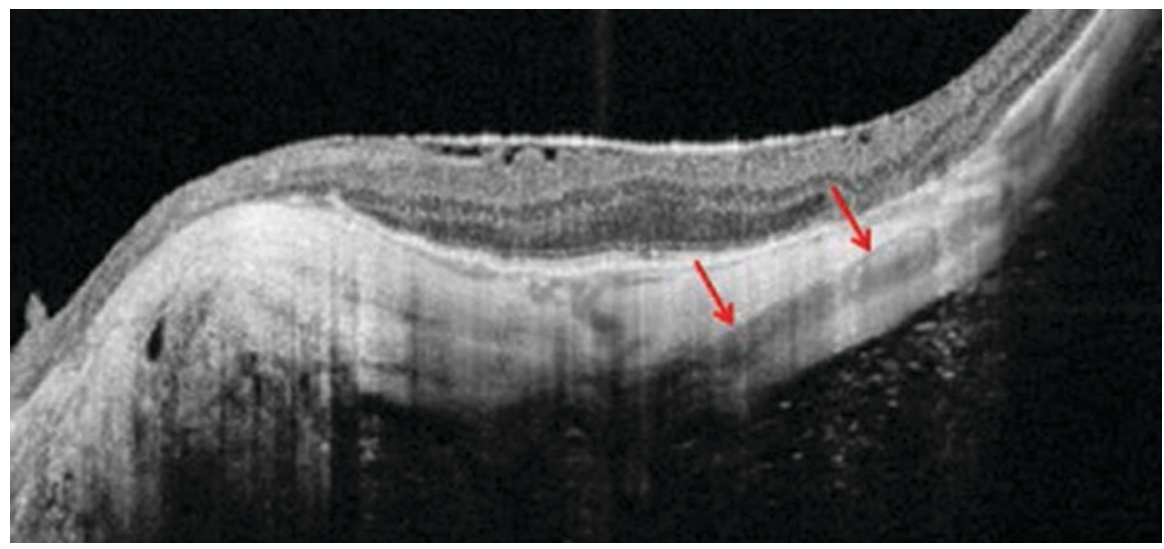

Fig. 11.2 Optical coherence tomographic image of the posterior sclera in a highly myopic eye showing the oblique course of a short posterior ciliary artery (red arrows) through the sclera

\section{References}

1. Heine L. Beiträge zur Anatomie des myopischen Auges. Arch Augenheilk. 1899;38:277-90.

2. Vurgese S, Panda-Jonas S, Jonas JB. Sclera thickness in human globes and its relations to age, axial length and glaucoma. PLoS One. 2012;7:e29692.

3. Jonas JB, Holbach L, Panda-Jonas S. Scleral cross section area and volume and axial length. PLoS One. 2014;9:e93551.

4. Shen L, Xu X, You QS, Gao F, Zhang Z, Li B, Jonas JB. Scleral thickness in Chinese eyes. Invest Ophthalmol Vis Sci. 2015;56:2720-7.

5. Shen L, You QS, Xu X, Gao F, Zhang Z, Li B, Jonas JB. Scleral and choroidal volume in relation to axial length in infants with retinoblastoma versus adults with malignant melanomas or end-stage glaucoma. Graefes Arch Clin Exp Ophthalmol. 2016;254:1779-86.

6. Wei WB, Xu L, Jonas JB, Shao L, Du KF, Wang S, Chen CX, Xu J, Wang YX, Zhou JQ, You QS. Subfoveal choroidal thickness: the Beijing Eye Study. Ophthalmology. 2013;120:175-80.

7. Jonas JB, Ohno-Matsui K, Jiang WJ, Panda-Jonas S. Bruch membrane and the mechanism of myopization. A new theory. Retina. 2017;37:1428-40.

8. Jonas JB, Holbach L, Panda-Jonas S. Bruch's membrane thickness in high myopia. Acta Ophthalmol. 2014;92:e470-4.

9. Wang X, Teoh CKG, Chan ASY, Thangarajoo S, Jonas JB, Girard MJA. Biomechanical properties of Bruch's membrane-choroid complex and their influence on optic nerve head biomechanics. Invest Ophthalmol Vis Sci. 2018;59:2808-17.

10. Jonas JB, Ohno-Matsui K, Holbach L, Panda-Jonas S. Retinal pigment epithelium cell density in relationship to axial length in human eyes. Acta Ophthalmol. 2017;95:e22-8.

11. Jonas JB, Xu L, Wei WB, Pan Z, Yang H, Holbach L, Panda-Jonas S, Wang YX. Retinal thickness and axial length. Invest Ophthalmol Vis Sci. 2016;57:1791-7.

12. Jonas RA, Wang YX, Yang H, Li JJ, Xu L, Panda-Jonas S, Jonas JB. Optic disc - fovea distance, axial length and parapapillary zones. The Beijing Eye Study 2011. PLoS One. 2015;10:e138701.

13. Guo Y, Liu LJ, Tang P, Feng Y, Wu M, Lv YY, Xu L, Jonas JB. Optic disc-fovea distance and myopia progression in school children: the Beijing Children Eye Study. Acta Ophthalmol. 2018. https://doi.org/10.1111/aos.13728. 
14. Jonas JB, Wang YX, Zhang Q, Liu Y, Xu L, Wei WB. Macular Bruch's membrane length and axial length. The Beijing Eye Study. PLoS ONE. 2015;10:e136833.

15. Jonas JB, Dichtl A. Optic disc morphology in myopic primary open-angle glaucoma. Graefes Arch Clin Exp Ophthalmol. 1997;235:627-33.

16. Xu L, Li Y, Wang S, et al. Characteristics of highly myopic eyes. The Beijing Eye Study. Ophthalmology. 2007;114:121-6.

17. Jonas JB, Berenshtein E, Holbach L. Lamina cribrosa thickness and spatial relationships between intraocular space and cerebrospinal fluid space in highly myopic eyes. Invest Ophthalmol Vis Sci. 2004;45:2660-5.

18. Jonas JB, Berenshtein E, Holbach L. Anatomic relationship between lamina cribrosa, intraocular space, and cerebrospinal fluid space. Invest Ophthalmol Vis Sci. 2003;44:5189-95.

19. Jonas JB, Jonas SB, Jonas RA, Holbach L, Dai Y, Sun X, Panda-Jonas S. Parapapillary atrophy: histological gamma zone and delta zone. PLoS One. 2012;7(10):e47237.

20. Dai Y, Jonas JB, Huang H, et al. Microstructure of parapapillary atrophy: beta zone and gamma zone. Invest Ophthalmol Vis Sci. 2013;54:2013-8.

21. Jonas JB, Wang YX, Zhang Q, Fan YY, Xu L, Wei WB, Jonas RA. Parapapillary gamma zone and axial elongation-associated optic disc rotation: The Beijing Eye Study. Invest Ophthalmol Vis Sci. 2016;57:396-402.

22. Fan YY, Jonas JB, Wang YX, Chen CX, Wei WB. Horizontal and vertical optic disc rotation. The Beijing Eye Study. PLoS One. 2017;12:e0175749.

23. Jonas JB, Ohno-Matsui K, Holbach L, Panda-Jonas S. Association between axial length and horizontal and vertical globe diameters. Graefes Arch Clin Exp Ophthalmol. 2017;255:237-42.

24. Jonas JB, Ohno-Matsui K, Spaide RF, et al. Macular Bruch's membrane holes in high myopia: associated with gamma zone and delta zone of parapapillary region. Invest Ophthalmol Vis Sci. 2013;54:1295-30.

25. You QS, Peng XY, Xu L, Chen CX, Wei WB, Wang YX, Jonas JB. Macular Bruch's membrane defects in highly myopic eyes. The Beijing Eye Study. Retina. 2016;36:517-23.

26. Ohno-Matsui K, Jonas JB, Spaide RF. Macular Bruch's membrane holes in choroidal neovascularization-related myopic macular atrophy by swept-source optical coherence tomography. Am J Ophthalmol. 2015;162:133-9.

27. Shinohara K, Tanaka N, Jonas JB, Shimada N, Moriyama M, Yoshida T, Ohno-Matsui $\mathrm{K}$. Ultrawide-field OCT to investigate relationships between myopic macular retinoschisis and posterior staphyloma. Ophthalmology. 2018. https://doi.org/10.1016/j.ophtha.2018.03.053.

28. Jonas JB, Gusek GC, Naumann GO. Optic disc, cup and neuroretinal rim size, configuration and correlations in normal eyes. Invest Ophthalmol Vis Sci. 1988;29:1151-8.

29. Na JH, Moon BG, Sung KR, Lee Y, Kook MS. Characterization of peripapillary atrophy using spectral domain optical coherence tomography. Korean J Ophthalmol. 2010;24:353-9.

30. Lee KY, Tomidokoro A, Sakata R, Konno S, Mayama C, Saito H, Hayashi K, Iwase A, Araie M. Cross-sectional anatomic configurations of peripapillary atrophy evaluated with spectral domain-optical coherence tomography. Invest Ophthalmol Vis Sci. 2010;51:666-71.

31. Park SC, De Moraes CG, Tello C, Liebmann JM, Ritch R. In-vivo microstructural anatomy of beta-zone parapapillary atrophy in glaucoma. Invest Ophthalmol Vis Sci. 2010;51:6408-13.

32. Manjunath V, Shah H, Fujimoto JG, Duker JS. Analysis of peripapillary atrophy using spectral domain optical coherence tomography. Ophthalmology. 2011;118:531-6.

33. Nonaka A, Hangai M, Akagi T, Mori S, Nukada M, Nakano N, Yoshimura N. Biometric features of peripapillary atrophy beta in eyes with high myopia. Invest Ophthalmol Vis Sci. 2011;52:6706-13.

34. Park SC, De Moraes CG, Teng CC, Tello C, Liebmann JM, Ritch R. Enhanced depth imaging optical coherence tomography of deep optic nerve complex structures in glaucoma. Ophthalmology. 2012;119:3-9.

35. Hayashi K, Tomidokoro A, Lee KY, Konno S, Saito H, Mayama C, Aihara M, Iwase A, Araie M. Spectral-domain optical coherence tomography of $\beta$-zone peripapillary atrophy: influence of myopia and glaucoma. Invest Ophthalmol Vis Sci. 2012;53:1499-505. 
36. Reis AS, Sharpe GP, Yang H, Nicolela MT, Burgoyne CF, Chauhan BC. Optic disc margin anatomy in patients with glaucoma and normal controls with spectral domain optical coherence tomography. Ophthalmology. 2012;119:738-47.

37. Jonas JB, Jonas SB, Jonas RA, Holbach L, Panda-Jonas S. Histology of the parapapillary region in high myopia. Am J Ophthalmol. 2011;152:1021-9.

38. Jonas JB, Holbach L, Panda-Jonas S. Peripapillary ring: histology and correlations. Acta Ophthalmol. 2014;92:e273-9.

39. Jonas JB, Jonas RA, Jonas SB, Panda-Jonas S. Lamina cribrosa thickness correlated with peripapillary sclera thickness. Acta Ophthalmol. 2012;90:e248-50.

40. Jonas JB, Jonas SB. Histomorphometry of the circular arterial ring of Zinn-Haller in normal and glaucomatous eyes. Acta Ophthalmol. 2010;88:e317-22.

41. Jonas JB, Holbach L, Panda-Jonas S. Peripapillary arterial circle of Zinn-Haller: location and spatial relationships. PLoS One. 2013;8:e78867.

42. Jonas JB, Papastathopoulos KI. Optic disc shape in glaucoma. Graefes Arch Clin Exp Ophthalmol. 1996;234:S167-73.

43. Guo Y, Liu LJ, Xu L, Lv YY, Tang P, Feng Y, Zhou JQ, Meng M, Jonas JB. Optic disc ovality in primary school children in Beijing. Invest Ophthalmol Vis Sci. 2015;56:4547-53.

44. Dai Y, Jonas JB, Ling Z, Sun X. Ophthalmoscopic-perspectively distorted optic disc diameters and real disc diameters. Invest Ophthalmol Vis Sci. 2015;56:7076-83.

45. Demer JL. Optic nerve sheath as a novel mechanical load on the globe in ocular ductionoptic nerve sheath constrains duction. Invest Ophthalmol Vis Sci. 2016;57:1826-38.

46. Wang X, Rumpel H, Lim WE, Baskaran M, Perera SA, Nongpiur ME, Aung T, Milea D, Girard MJ. Finite element analysis predicts large optic nerve head strains during horizontal eye movements. Invest Ophthalmol Vis Sci. 2016;57:2452-62.

47. Jonas JB, Dai Y, Panda-Jonas S. Peripapillary suprachoroidal cavitation, parapapillary gamma zone and optic disc rotation due to the biomechanics of the optic nerve dura mater. Invest Ophthalmol Vis Sci. 2016;57:4373.

48. Wang X, Rumpel H, Lim WE, Baskaran M, Perera SA, Nongpiur ME, Aung T, Milea D, Girard MJ. Author Response: Peripapillary suprachoroidal cavitation, parapapillary gamma zone and optic disc rotation due to the biomechanics of the optic nerve dura mater. Invest Ophthalmol Vis Sci. 2016;57:4374-5.

49. Ohno-Matsui K, Shimada N, Akiba M, Moriyama M, Ishibashi T, Tokoro T. Characteristics of intrachoroidal cavitation located temporal to optic disc in highly myopic eyes. Eye. 2013;27:630-8.

50. Dai Y, Jonas JB, Ling Z, Wang X, Sun X. Unilateral peripapillary intrachoroidal cavitation and optic disc rotation. Retina. 2015;35:655-9.

51. Jonas JB. Optic disc size correlated with refractive error. Am J Ophthalmol. 2005;139:346-8.

52. Xu L, Wang YX, Wang S, Jonas JB. Definition of high myopia by parapapillary atrophy. The Beijing Eye Study. Acta Ohthalmol. 2010;88:e350-1.

53. Xu L, Wang Y, Wang S, Wang Y, Jonas JB. High myopia and glaucoma susceptibility. The Beijing Eye Study. Ophthalmology. 2007;114:216-20.

54. Liu HH, Xu L, Wang YX, Wang S, You QS, Jonas JB. Prevalence and progression of myopic retinopathy in Chinese adults: The Beijing Eye Study. Ophthalmology. 2010;117:1763-8.

55. Nakazawa M, Kurotaki J, Ruike H. Longterm findings in peripapillary crescent formation in eyes with mild or moderate myopia. Acta Ophthalmol. 2008;86:626-9.

56. Podos S, Becker B, Mortion W. High myopia and primary open-angle glaucoma. Am J Ophthalmol. 1966;62:1039-43.

57. Greve EL, Furuno F. Myopia and glaucoma. Graefes Arch Clin Exp Ophthalmol. 1980;213:33-41.

58. Daubs JG, Crick RP. Effect of refractive error on the risk of ocular hypertension and open angle glaucoma. Trans Ophthalmol Soc Aust. 1981;101:121-6.

59. Phelps CD. Effect of myopia on prognosis in treated primary open-angle glaucoma. Am J Ophthalmol. 1982;93:622-8. 
60. Perkins ES, Phelps CD. Open angle glaucoma, ocular hypertension, low-tension glaucoma, and refraction. Arch Ophthalmol. 1982;100:1464-7.

61. Leske MC, Connell AM, Wu SY, Hyman LG, Schachat AP. Risk factors for open-angle glaucoma. The Barbados Eye Study. Arch Ophthalmol. 1995;113:918-24.

62. Chihara E, Liu X, Dong J, Takashima Y, Akimoto M, Hangai M, Kuriyama S, Tanihara H, Hosoda M, Tsukahara S. Severe myopia as a risk factor for progressive visual field loss in primary open-angle glaucoma. Ophthalmologica. 1997;211:66-71.

63. Mitchell P, Hourihan F, Sandbach J, Wang JJ. The relationship between glaucoma and myopia: the Blue Mountains Eye Study. Ophthalmology. 1999;106:2010-5.

64. Grodum K, Heijl A, Bengtsson B. Refractive error and glaucoma. Acta Ophthalmol Scand. 2001;79:560-6.

65. Jonas JB, Martus P, Budde WM. Anisometropia and degree of optic nerve damage in chronic open-angle glaucoma. Am J Ophthalmol. 2002;134:547-51.

66. Wong TY, Klein BE, Klein R, Knudtson M, Lee KE. Refractive errors, intraocular pressure, and glaucoma in a white population. Ophthalmology. 2003;110:211-7.

67. Leske MC, Heijl A, Hussein M, Bengtsson B, Hyman L, Komaroff E, Early Manifest Glaucoma Trial Group. Factors for glaucoma progression and the effect of treatment: the early manifest glaucoma trial. Arch Ophthalmol. 2003;121:48-56.

68. Jonas JB, Budde WM. Optic nerve damage in highly myopic eyes with chronic open-angle glaucoma. Eur J Ophthalmol. 2005; 15:41-7.

69. Kuzin AA, Varma R, Reddy HS, Torres M, Azen SP, Los Angeles Latino Eye Study Group. Ocular biometry and open-angle glaucoma: the Los Angeles Latino Eye Study. Ophthalmology. 2010;117:1713-9.

70. Nagaoka N, Jonas JB, Morohoshi K, Moriyama M, Shimada N, Yoshida T, Ohno-Matsui K. Glaucomatous-type optic discs in high myopia. PLoS One. 2015;10:e138825.

71. Jonas JB, Weber P, Nagaoka N, Ohno-Matsui K. Glaucoma in high myopia and parapapillary delta zone. PLoS One. 2017;12:e0175120.

72. Balaratnasingam C, Morgan WH, Johnstone V, et al. Histomorphometric measurements in human and dog optic nerve and an estimation of optic nerve pressure gradients in human. Exp Eye Res. 2009;89:618-28.

73. Morgan WH, Yu DY, Balaratnasingam CX. The role of cerebrospinal fluid pressure in glaucoma pathophysiology: the dark side of the optic disc. J Glaucoma. 1998;17:408-13.

74. Berdahl JP, Allingham RR, Johnson DH. Cerebrospinal fluid pressure is decreased in primary open-angle glaucoma. Ophthalmology. 2008;115:763-8.

75. Ren R, Jonas JB, Tian G, Zhen Y, Ma K, Li S, Wang H, Li B, Zhang X, Wang N. Cerebrospinal fluid pressure in glaucoma. A prospective study. Ophthalmology. 2010;117:259-66.

76. Jonas JB, Wang N, Yang D, Ritch R, Panda-Jonas S. Facts and myths of cerebrospinal fluid pressure for the physiology of the eye. Prog Retin Eye Res. 2015;46:67-83.

77. Burgoyne CF, Morrison JC. The anatomy and pathophysiology of the optic nerve head in glaucoma. J Glaucoma. 2001;10:S16-8.

78. Dichtl A, Jonas JB, Naumann GO. Histomorphometry of the optic disc in highly myopic eyes with absolute secondary angle closure glaucoma. Br J Ophthalmol. 1998;82:286-9.

79. Hayreh SS. Blood supply of the optic nerve head and its role in optic atrophy, glaucoma, and oedema of the optic nerve. Br J Ophthalmol. 1969;53:721-48.

80. Jonas JB, Xu L, Zhang L, Wang Y, Wang Y. Optic disk size in chronic glaucoma. The Beijing Eye Study. Am J Ophthalmol. 2006;142:168-70.

81. Wang YX, Hu LN, Yang H, Jonas JB, Xu L. Frequency and associated factors of structural progression of open-angle glaucoma in the Beijing Eye Study. Br J Ophthalmol. 2012;96:811-5.

82. Jiang R, Wang YX, Wei WB, Xu L, Jonas JB. Peripapillary choroidal thickness in adult Chinese: The Beijing Eye Study. Invest Ophthalmol Vis Sci. 2015;56:4045-52.

83. Jonas JB, Nagaoka N, Fang YX, Weber P, Ohno-Matsui K. Intraocular pressure and glaucomatous optic neuropathy in high myopia. Invest Ophthalmol Vis Sci. 2017;58:5897-906. 
Open Access This chapter is licensed under the terms of the Creative Commons Attribution 4.0 International License (http://creativecommons.org/licenses/by/4.0/), which permits use, sharing, adaptation, distribution and reproduction in any medium or format, as long as you give appropriate credit to the original author(s) and the source, provide a link to the Creative Commons license and indicate if changes were made.

The images or other third party material in this chapter are included in the chapter's Creative Commons license, unless indicated otherwise in a credit line to the material. If material is not included in the chapter's Creative Commons license and your intended use is not permitted by statutory regulation or exceeds the permitted use, you will need to obtain permission directly from the copyright holder. 